\title{
Pharmacogenetic testing of CYP2C9 and VKORC1 alleles for warfarin
}

David A. Flockhart, MD, $P h D^{1}$, Dennis O'Kane, $P h D^{2}$, Marc S. Williams, $M D^{3}$, and Michael S. Watson, $P h D^{4}$ On Behalf of the ACMG Working Group on Pharmacogenetic Testing of CYP2C9, VKORC1 Alleles for Warfarin Use: David A. Flockhart, $M D, P h D^{1}$, Brian Gage, $M D^{5}$, Roy Gandolfi, $M D^{3}$, Richard King, $M D^{6}$, Elaine Lyon, $P h D^{7}$, Robert Nussbaum, $M D^{8}$, Dennis O'Kane, PhD' ${ }^{2}$, Kevin Schulman, $M D^{9}$, David Veenstra, $P h D^{10}$, Marc S. Williams, $M D^{3}$, and Michael S. Watson, $P h D^{4}$

Key Words: pharmacogenetics, warfarin, coumadin, CYP2CP, VKORC1, thromboembolism, hypercoagulation

Disclaimer: American College of Medical Genetics statements and guidelines are designed primarily as an educational resource for medical geneticists and other health care professionals to help them provide quality medical genetic services. Adherence to these standards and guidelines does not necessarily ensure a successful medical outcome. These statements and guidelines should not be considered inclusive of all proper procedures and tests or exclusive of other procedures and tests that are reasonably directed to obtaining the same results. In determining the propriety of any specific procedure or test, the health care professional should apply his or her own professional judgment to the specific clinical circumstances presented by the individual patient or specimen. It may be prudent, however, to document in the patient's record the rationale for any significant deviation from these standards and guidelines.

\begin{abstract}
Warfarin (Coumadin) is a potent drug that when used judiciously and monitored closely, leads to substantial reductions in morbidity and mortality from thromboembolic events. However, even with careful monitoring, initiation of warfarin dosing is associated with highly variable responses between individuals and challenges achieving and maintaining levels within the narrow therapeutic range that can lead to adverse drug events. Variants of two genes, CYP2C9 and VKORC1, account for 30-50\% of the variability in dosing of warfarin; thus, many believe that testing of these genes will aid in warfarin dosing recommendations. Evidence about this test is evolving rapidly, as is its translation into clinical practice. In an effort to address this situation, a multidisciplinary expert group was organized in November 2006 to evaluate the role of CYP2C9 and VKORC1 testing in altering warfarin-related therapeutic goals and reduction of adverse drug events. A recently completed Rapid-ACCE (Analytical, Clinical Validity, Clinical Utility, and Ethical, Legal, and Social Implications) Review, commissioned to inform this work group, was the foundation for this analysis. From this effort, specific recommendations for the appropriate use of CYP2C9 and VKORC1 testing were developed and are presented here. The group determined that the analytical validity of these tests has been met, and there is strong evidence to support association between these genetic variants and therapeutic dose of warfarin. However, there is insufficient evidence, at this time, to recommend for or against routine CYP2C9 and VKORC1 testing in warfarin-naive patients. Prospective clinical trials are needed that provide direct evidence of the benefits, disadvantages, and costs associated with this testing in the setting of initial warfarin dosing. Although the routine use of warfarin genotyping is not endorsed by this work group at this time, in certain situations, CYP2C9 and VKORC1 testing may be useful, and warranted, in determining the cause of unusual therapeutic responses to warfarin therapy. Genet Med 2008:10(2):139-150.
\end{abstract}

There is perhaps no drug whose therapeutic range is functionally as narrow as that of warfarin. Warfarin has a very large marketplace with over 30 million prescriptions in the United
States in 2004, including up to a million new patients initiated on therapy each year. Although effective in reducing thrombotic events, warfarin's use is associated with 800 reports to the
Michael S. Watson, PhD, FACMG, Executive Director, American College of Medical Genetics, American College of Medical Genetics Foundation, 9650 Rockville Pike, Bethesda, MD 20814-3998.E-mail:mwatson@acmg.net

Submitted for publication November 29, 2007.

Accepted for publication November 30, 2007. DOI: 10.1097/GIM.0b013e318163c35f 
Food and Drug Administration (FDA) of adverse drug events leading to an emergency room visit each year in the United States; though reports in 2005 indicated that emergency room visits numbered as many as $36 .{ }^{1}$ It is widely recognized that dosing of warfarin is difficult, and this has resulted in the development over the last 20 years of the International Normalized Ratio (INR) monitoring system. The INR is routinely reported as the ratio of the patient's prothrombin time to that of a reference population, corrected for the sensitivity of the thromboplastin reagent used. This also led to the creation of clinics dedicated to the treatment and monitoring of patients taking warfarin. Warfarin dosing is typically adjusted to maintain the INR at $2.5 \pm 0.5$ and at $3.0 \pm 0.5$ for higher risk patients, including those with certain mechanical heart valves. Efforts to improve the utility of the INR have been ongoing for more than 25 years. While the intensity of this cumulative effort by many health care professionals reflects our collective concern that warfarin be used as safely and effectively as possible, it also makes clear the difficulty of doing so, and implicitly suggests the value of improved means of monitoring. That said, any technology that purports to improve our ability to use warfarin safely and effectively must be evaluated in a clinical context where the existing clinical systems designed to do so are already effectively in place. We present here the results of a recent assessment of the evidence surrounding the use of pharmacogenetic testing as a means of improving the safety and efficacy of warfarin use. The purpose of this project was 2-fold: (1) to identify and evaluate the best-available evidence and the gaps therein for the use of pharmacogenetic testing to guide warfarin use. To inform a multidisciplinary expert group in its evaluation of the scientific and clinical literature, a Rapid-ACCE (Analytical, Clinical Validity, Clinical Utility, and Ethical, Legal, and Social Implications) Review of the evidence base was commissioned; and (2) to offer recommendations on whether the evidence supports the use of testing of CYP2C9 and VKORC1 as a standard of care. Further, it is to provide guidance to improve and maintain the high quality of testing for warfarin sensitivity should this testing be requested by the patient or their health care provider. It is anticipated that this document and its guidance will be updated as outcomes from prospective studies become available.

\section{The clinical value of warfarin}

Warfarin is indicated for the prophylaxis and treatment of venous thrombosis and pulmonary embolism, for thromboembolic complications associated with atrial fibrillation and cardiac valve replacement, and in the postmyocardial infarction setting where it is used to reduce the risk of death, recurrent myocardial infarction, and thromboembolic events. In all these settings it is widely recognized that warfarin is effective (no studies are available that address the question of whether CYP2C9 or VKOR genotypes retrospectively or prospectively predict the risk of thrombosis or myocardial infarction in patients), ${ }^{2-4}$ but underutilized because of its toxicity. ${ }^{5,6}$

\section{Warfarin and dosing variability}

Warfarin dosing is highly variable between individuals. A number of factors affect warfarin dosing, including nongenetic factors (drug-drug interactions, environmental factors, including diet, alcohol consumption, and smoking) and genetic factors. There is ample evidence that genetic factors explain about $40 \%$ of warfarin dosing variability. ${ }^{1,7-9}$

Two generalized clinical scenarios exist for warfarin dosing: warfarin resistance and warfarin sensitivity. Warfarin resistance is an infrequently encountered clinical scenario that may be due to mutations in the vitamin $\mathrm{K}$ epoxide reductase complex 1 gene (VKORC1), a recently identified target of warfarin inhibition (OMIM 608547). ${ }^{10}$ These mutations make VKORC1 less susceptible to warfarin inhibition. Heterozygosity leads to increased warfarin requirements, generally in excess of $80 \mathrm{mg} /$ week to maintain appropriate anticoagulation status. ${ }^{11}$ Complete inactivation of VKORC1 causes a rare syndrome of multiple coagulation factor defects (combined deficiency of vitamin k-dependent clotting factors, type 2; OMIM 607473 ) that is responsive to oral administration of vitamin $\mathrm{K}$.

The more frequent clinical scenario is warfarin sensitivity. In this context, warfarin is used for anticoagulation to produce an increase in the prothrombin time, as expressed by the INR, to between 2 and 3 (or between 2.5 and 3.5 for some artificial heart valves). Some individuals are sensitive to warfarin, requiring decreased dosing; otherwise, the INR may increase significantly above their target range. Major bleeding episodes, including cerebrovascular bleeds, are significant adverse events leading to morbidity and mortality. These are associated with warfarin sensitivity and INR $>5$.

\section{Genes and polymorphisms that are independent predictors of warfarin dosing variability}

Genetic variability of warfarin dosing has been attributed to polymorphisms in genes that encode the enzymes that metabolize warfarin, the targets of warfarin inhibition in the vitamin K cycle, the gene responsible for vitamin K-dependent $\gamma$-carboxylation of proteins, and polymorphisms within coagulation factors.

Several genes are involved in the metabolism of warfarin, ${ }^{12} \mathrm{a}$ racemic mixture of S- and R-warfarin enantiomers. S-warfarin, the more active isomer, is metabolized predominately by CYP2C9 (OMIM 601130). The Human Cytochrome P450 (CYP) Allele Nomenclature Committee recognizes 37 alleles of CYP2C9 (http://www.cypalleles.ki.SE/cyp2c9.htm). More than 300 variations in DNA sequence have been reported. However, the functional impact of many of these polymorphisms is not well established. Using the allele designations from the pharmacogenetics literature, $C Y P 2 C 9^{*} 2$ and $C Y P 2 C 9^{*} 3$ are clearly the major polymorphic alleles with sufficient evidence linking them to altered warfarin metabolism. R-warfarin, which has $20-30 \%$ of the anticoagulation effect of S-warfarin, is metabolized by a number of CYP450 enzymes, including CYP1A2 (OMIM 124060), CYP2C8 (OMIM 601129), CYP2C19 (OMIM 124020), CYP3A4 (OMIM 124010), and CYP3A5 (OMIM 605325). All of the genes for these enzymes have polymorphic 
variations that may affect metabolic activity. However, the variable metabolism of S-warfarin, the more effective isomer in anticoagulation, is believed responsible for much if not most of the variability in warfarin dosing. The major pharmacokinetic change associated with deficient CYP2C9 is that the halflife of bioactive S-warfarin is increased. ${ }^{13}$ This will increase the time to reaching steady-state concentrations of warfarin. Accordingly, the INR determined at a set time in a protocol may not reflect the final steady-state INR on the warfarin dose used for the patient who is heterozygous or homozygous for CYP2C9 polymorphisms. This could result in increases in the INR above what would be predicted based on nongenetic factors alone.

CYP2C9 ${ }^{*} 2$ and $C Y P 2 C 9^{*} 3$ have several subtypes depending upon single-nucleotide polymorphisms (SNPs) in the upstream $5^{\prime}$-noncoding region of the gene, producing distinct haplotypes. However, these upstream polymorphisms are not significant independent determinants of warfarin dose variability. ${ }^{14} \mathrm{CYP} \mathrm{C}^{\star} 2$ and ${ }^{*} 3$ each have defining coding SNPs (cSNPs) that alter the amino acid sequence of the proteins and result in altered enzymatic activity.

VKORC1 is the major target of warfarin inhibition. The VKORC1 gene has only recently been identified along with polymorphisms that result in pharmacodynamic effects on warfarin. VKORC1 functions to convert vitamin K-epoxide, formed during the carboxylation of several proteins, to the vitamin K-quinone, allowing the vitamin K cycle to function catalytically in the generation of $\gamma$-carboxyglutamyl residues in proteins, including clotting factors. Relative deficiency of VKORC1 results in less $\gamma$-carboxylation of these proteins and the production of clotting factors that are deficient in coagulation activity.

$\gamma$-Glutamyl carboxylase (OMIM 137167) is responsible for posttranslational modification of glutamyl residues in proteins, including several coagulation factors. Deficiency of $\gamma$-glutamyl residues in the procoagulant clotting factors results in factors that are less effective in coagulation and leads to increased bleeding times with higher INRs. The procoagulant factors affected by warfarin inhibition of carboxylation are factors II, VII, IX, X, and the anticoagulant factors affected are Protein S and Protein C. While all of these genes and their encoded proteins may impact aspects of warfarin dosing in individual patients, contributing to variation in warfarin dose requirements, at this time there is limited evidence to support testing for only two genes and a limited number of polymorphisms that impact warfarin dosing in a significant number of patients. There are few data available to guide the interpretation of findings of other variants.

\section{PURPOSE}

This position statement is intended as an educational resource. The purpose of this document is to evaluate the use of genetic testing to inform warfarin dosing, using best evidence to determine whether a clinical guideline should be developed. Limitations in the evidence in at least one critical area led to this document being structured as a summary of current knowledge with the identification of gaps in knowledge. Al- though it was not considered appropriate to argue that pharmacogenetic testing to inform warfarin dosing be considered a standard of care, the document does provide guidance on pharmacogenetic testing for warfarin sensitivity to improve and maintain the high quality of testing during this clinical investigative phase, and to make it possible for providers that choose to order testing to use the results to make patient management decisions based on best-available evidence and to effectively communicate results to patients. It highlights current practices and therapeutic approaches related to the dosing and use of warfarin and the evidence that surrounds the use of emerging pharmacogenetic tests to improve dosing to minimize severe outcomes related to warfarin use.

\section{METHODS/PROCESS}

\section{Consensus development panel}

A multidisciplinary group of experts in clinical pharmacology, genetic testing, and clinical genetics services, evidencebased medicine, community-based anticoagulation services, health policy, regulation, and financing was assembled to review the evidence base and develop management guidelines. A Rapid-ACCE Review ${ }^{15}$ was commissioned to identify and evaluate the evidence base with a focus on the quality of the data and the identification of gaps in the evidence. The RapidACCE Review process that was used here ${ }^{15}$ was designed to speed up reviews at lower cost as compared with either full ACCE Reviews ${ }^{16}$ or other similarly expensive systems when there is a relatively small evidence base for a targeted review. The published literature is supplemented with data from the gray literature that may include unpublished laboratory-based data on performance characteristics of particular tests offered by individual laboratories, FDA submissions, laboratory Web site information, abstracts, and materials distributed at meetings. It provided the evidence base on which the experts reached consensus on the recommendations herein. The main focus of the review was on evidence regarding the efficacy of identifying variant genotypes whose detection might minimize the more frequent adverse clinical scenario of serious bleeding. Although thrombotic events are also a consequence of the narrow therapeutic range of warfarin, the evidence review was focused on the hemorrhagic events. The ACCE Review is based on 44 questions that assess the availability, quality, and usefulness of existing data on DNA-based tests and testing algorithms. The questions are divided between four areas of evidence: (1) analytic validity encompassing the preanalytic, analytic, and postanalytic phase of testing, (2) clinical validity, (3) clinical utility that assesses the risks and benefits of testing, and (4) the ethical, legal, and social implications of the testing. The ACCE Review considered evidence that was available as of November, 2006.

A full-evidence evaluation had been planned initially to correlate the strength of the evidence to the recommendations. However, because of the absence of evidence in critical areas of the review, the decision was made to present the information so as to highlight the gaps in the evidence base to inform a broader research agenda that can develop the appropriate data. 
Table 1

CYP2C9

\begin{tabular}{ll}
\hline Genotypes & Impact on warfarin metabolism \\
${ }^{*} 1 /{ }^{*} 1$ & Extensive (normal) metabolism \\
${ }^{*} 1 /{ }^{* 2}$ & Intermediate metabolism \\
${ }^{*} 1 /{ }^{* 3}$ & Slow metabolism \\
${ }^{*} 2 /{ }^{* 2}$ & \\
${ }^{*} 2 /{ }^{* 3}$ & \\
${ }^{*} 3 /{ }^{* 3}$ & \\
\hline
\end{tabular}

Conflict of interest statements were provided by the participants. All members of the panel reviewed and approved the final statement. Consensus was defined as agreement among all members of the panel. Penultimate drafts of these guidelines were shared with an external review group consisting of individuals with expertise similar to those of the work group but who were not directly involved in the group's deliberations. Their suggestions were considered by the expert work group, and changes were made as considered appropriate by this group.

\section{Nomenclature}

For the purposes of this document, the nomenclature shown in Table 1 that describes the impact of CYP2C9 and VKORC1 alleles on warfarin metabolism is used.

For $V K O R C 1$, the five clinically relevant variants are in strong linkage disequilibrium in non-Hispanic whites. The associated haplotypes ( $\mathrm{AA}, \mathrm{AB}$, and $\mathrm{BB}$ ) are used to describe the alleles (Table 2; the allele we have designated as the $\mathrm{B}$ allele is also referred to as the $\mathrm{G}$ allele).

\section{Target audience}

This guideline is directed at a wide range of providers. Although care is commonly provided by clinical pharmacologists, pharmacists and hematologists, primary care providers, those involved in anticoagulation clinics, and other specialists are often involved in the prescribing and dosage adjustments of warfarin. Medical geneticists are more likely to be involved in the laboratory testing and clinical decision support that accompanies testing. The identification of gaps in the literature is directed at clinical investigators.

\section{Analytical validity}

The bulk of the literature focuses on the more frequent genotypes of CYP2C9 and VKORC1. For CYP2C9, most data re-

Table 2

VKORC1

\begin{tabular}{lc}
\hline Haplotypes & Impact on warfarin metabolism \\
\hline AA & High sensitivity \\
AB & Medium sensitivity \\
BB & Low sensitivity \\
\hline
\end{tabular}

Table 3

VKORC1 alleles

\begin{tabular}{lll}
\hline Allele & \multicolumn{1}{c}{ Variant } & Activity \\
\hline${ }^{*} 1$ & Wild type & Normal \\
${ }^{*} 2$ & R144C $(3608 \mathrm{C} \rightarrow \mathrm{T})$ & Decreased \\
$\star 3$ & I359L $(42614 \mathrm{~A} \rightarrow \mathrm{C})$ & Decreased \\
${ }^{*} 4$ & I359T $(42615 \mathrm{~T} \rightarrow \mathrm{C})$ & Decreased \\
${ }^{*} 5$ & D360E, $42619 \mathrm{C} \rightarrow \mathrm{G}$ & Decreased \\
${ }^{*} 6$ & 10601delA $(818 \mathrm{delA})$ & Null \\
\hline
\end{tabular}

late to the wild-type CYP2C9 allele $\left({ }^{*} 1\right)$ and two of the many variants $\left({ }^{\star} 2\right.$ and $\left.{ }^{*} 3\right)$ that are associated with reduced metabolism of warfarin. Other alleles are rare. The gene variants that define the alleles are shown in Table 3.

For VKORC1, most literature focuses on five gene variants, $-1639 \mathrm{G} \rightarrow \mathrm{A}, 1173 \mathrm{C} \rightarrow \mathrm{T}, 1542 \mathrm{G} \rightarrow \mathrm{C}, 2255 \mathrm{~T} \rightarrow \mathrm{C}$, and $3730 \mathrm{G} \rightarrow$ A. The functional variation identified in $V K O R C 1$ is the promoter $-1639 \mathrm{G} \rightarrow \mathrm{A}$. Individuals with the $-1639 \mathrm{~A}$ allele express less VKORC1 enzyme. Additional SNPs, such as $1173 \mathrm{C} \rightarrow \mathrm{T}$, $1542 \mathrm{G} \rightarrow \mathrm{C}, 2255 \mathrm{~T} \rightarrow \mathrm{C}$, and $3730 \mathrm{G} \rightarrow \mathrm{A},{ }^{17}$ are in linkage disequilibrium with the $-1639 \mathrm{G} \rightarrow$ A polymorphism and permit defining haplotypes. However, most in vitro diagnostic test manufacturers are including testing involving the $-1639 \mathrm{G} \rightarrow$ A or the $1173 \mathrm{C} \rightarrow$ T polymorphisms. As such, analytical validity data largely relate to testing of these alleles. If additional polymorphisms are included in the test panel, they need to be validated analytically as well as clinically.

There are many generic analytical methodologies for the identification of the alleles of interest. As with other genetic tests, most data are based on testing performed with anticoagulated whole blood as the specimen. Other specimen types, including cells from buccal mucosa, should be validated for performance in genotyping assays. Attention should be paid to DNA extraction and the resulting DNA concentration needed for accurate testing. Alternative methods using whole-genome amplified DNA also must be thoroughly validated to ensure accurate testing.

Few genetic tests that arise as laboratory-developed tests have had extensive comparisons of analytical performance as occurs when manufacturers develop devices and kits for genetic testing. Based on generic evidence from molecular tests, analytical sensitivity and specificity for CYP2C9 testing are expected to be above $98 \%$ for the former and $99.5 \%$ for the latter. ${ }^{18,19}$ Specific data on VKORC1 testing are quite limited although there is nothing in the assays that would suggest the likelihood of differences in analytical performance from that of other tests. In fact, most analytical performance data are held by individual laboratories that offer specific tests where they are documented in laboratory notebooks for laboratory inspectors to evaluate. Laboratories should follow standard practices to maximize the analytical performance of their tests.

Guidance, Standards, Policies, and Checklist documents are available from a number of organizations that pertain, at least in part, to warfarin sensitivity testing. These include "Stan- 
dards and Guidelines for Clinical Genetics Laboratories" (American College of Medical Genetics), 20 "Molecular Diagnostic Methods for Genetic Diseases" (Clinical Laboratory Standards Institute), ${ }^{21}$ "Laboratory Standards, sections 1 and 5-9" (New York State Department of Health), "Molecular Pathology Checklist" (College of American Pathologists), and Organization for Economic Co-Operation and Development Guidelines for Quality Assurance in Molecular Genetic Testing. ${ }^{22}$ In addition, a draft recommendation for pharmacogenetic testing, "Guidelines and Recommendations for Laboratory Analysis and Application of Pharmacogenetics to Clinical Practice" is available from the National Academy of Clinical Biochemistry. ${ }^{23}$ There is no intention to reiterate or reproduce these standards and recommendations in the following discussion.

\section{Gaps in evidence}

CYP2C9 and VKORC1 testing arose as laboratory-developed tests as many genetic tests do. In the absence of FDA-cleared manufactured devices during the clinical investigative stage, most data on analytical performance are held by the laboratories that develop and offer the testing. Such data are infrequently published but are available through the gray literature via direct contact with laboratories that have documented their test performances. As such, the major gaps in the evidence base surrounding analytical performance of CYP2C9 and VKORC1 testing involve the availability of data from individual laboratories offering the tests. As with many genetic tests, there is a mix of rare and common molecular targets of testing. This leads to:

- a poorly organized evidence base related to the analytical performance of tests targeting the rarer variants;

- limited information on the performance of clinical laboratories, though this may be improved with the advent of a College of American Pathologists (CAP)/American College of Medical Genetics proficiency testing program for warfarin pharmacogenetic testing that should begin to provide data on concordance of testing results, sample types tested, internal test validation, turn-around-time, and result report content; and

- lack of comparative information on the performance of the multiple laboratory methods used for testing.

There are also gaps in knowledge of INR testing related to:

- intralaboratory performance differences;

- differences between point-of-care and clinical laboratorybased testing; and

- direct comparison of the utility of the INR as compared with molecular testing.

\section{Recommendations}

In the absence of extensive experience in testing of CYP2C9 and, particularly, $V K O R C 1$ testing laboratories should:

- maximize the performance of their testing methods

- confirm results with independent methods

- monitor test failure rates to allow for the identification of problems that alter historical trends in test performance
It is also recommended that:

- the VKORC1 $-1639 \mathrm{G} \rightarrow \mathrm{A}$ or the $1173 \mathrm{C} \rightarrow \mathrm{T}$ variants be tested

- defined haplotypes that incorporate additional polymorphisms may be used if the assays are validated. Testing of multiple variants in tight linkage disequilibrium can be redundant, leading to increased cost with limited benefit. It should be made clear that presumed haplotypes are based on the population data available at the time

- data on performance should be associated with clinical data in a centralized repository such as PharmGKB

- laboratories should participate in the proficiency testing program for pharmacogenetics sponsored by the College of American Pathologists and the American College of Medical Genetics.

\section{Clinical validity and utility of pharmacogenetic testing to reduce adverse bleeding events during warfarin dosing}

The clinical validity and utility of pharmacogenetic tests hinge on their analytical validity, but also depend upon how well they impact important clinical outcomes of the drug's effect. In this respect, pharmacogenetic tests differ from conventional genetic tests designed to detect a disease in four important ways. First, all drugs have side effects. It follows that a pharmacogenetic test may be designed to predict efficacy or to predict adverse events, or both. Because a given inherited genetic variant may influence the effect of a drug on a given patient in more than one way, it is not always possible to use a single phenotype as a measure of clinical validity or utility. Second, pharmacogenetic tests may be used to predict a drug's dose or to predict the amount of time that a patient will stay within the therapeutic range. Dose and time are linear variables in contrast to the dichotomous presence or absence of disease. Tests designed to assess the specificity or sensitivity of a dichotomous variable are not designed to assess the ability of a test to predict a linear variable like dose or time to steady state, and other statistical approaches may therefore be necessary. Third, pharmacogenetic tests fundamentally involve a drug as well as a disease that drug is being used to treat or prevent and it follows that a test's utility in a particular patient is inextricably linked to the drug's utility in that patient. Fourth, pharmacogenetic tests may be used in large numbers of patients, in contrast to genetic testing for rare genetic disease. In the context of this report, pharmacogenetic testing to predict the adverse effects of warfarin has the potential to be carried out on hundreds of thousands of people who begin to take warfarin every year. The requirement for robust, repeatable testing and for clinical validity and utility of the test results is therefore high.

\section{Associating pharmacogenetic variants with warfarin dose}

As indicated above, two common SNPs in the CYP2C9 system are associated with impaired metabolism of warfarin, whereas SNPs in the gene for VKORC1 correlate with warfarin sensitivity and resistance. 
Since the first study demonstrating an association between warfarin dose and CYP2C9 variants in an anticoagulation clinic in 1999, ${ }^{24}$ a large number of studies have shown strong associations between these variants and the ultimate steadystate dose of warfarin. These studies have been replicated in diverse settings and involve large numbers of patients with different indications for treatment as well as of different ethnicities, including a significant number of Asian and African Americans. The contribution of these variants to the variability in dose at steady state has been estimated, from these studies and they have consistently shown that both CYP2C9 and VKORC1 variants are associated with dose. This relationship persists when other clinical parameters such as the first warfarin dose, age, and smoking status are included. ${ }^{25}$

\section{Cytochrome P450 2C9 (OMIM 601130)}

The defining $\mathrm{cSNP}$ for $\mathrm{CYP} 2 \mathrm{C} 9^{*} 2$ is the $430 \mathrm{C} \rightarrow \mathrm{T}$ transition that produces the amino acid change $\mathrm{R} 144 \mathrm{C}$. This amino acid substitution decreases enzymatic activity and more importantly decreases the intrinsic clearance compared with the enzyme encoded by unaltered reference sequence (designated ${ }^{\star} 1$ ). Changes in intrinsic clearance can be described in the context of the expected percent reduction in dose required to achieve the same effect. Heterozygosity for $C Y P 2 C 9^{*} 2$ decreases the dose for S-warfarin to $78 \%$ of that found for homozygous ${ }^{*} 1$ individuals $\left({ }^{\star} 1 /{ }^{\star} 1\right)$. Homozygosity for ${ }^{\star} 2$ decreases the dose to $57 \%$ that of the homozygous ${ }^{\star} 1$ individuals. ${ }^{1}$

The defining cSNP for $\mathrm{CYP}_{2} \mathrm{C}^{\star} 3$ is a transversion at $1075 \mathrm{~A} \rightarrow \mathrm{C}$ that produces the amino acid substitution I359L. This amino acid substitution decreases enzymatic activity by $95 \%$ compared with the enzyme encoded by the unaltered ${ }^{\star} 1$ reference sequence. Heterozygosity for $C Y P 2 C 9^{*} 3$ would lead to a decrease in the dose of warfarin to $66 \%$ of that required for homozygous ${ }^{\star} 1$ individuals. Homozygosity for ${ }^{\star} 3$ decreases the dose to $24 \%$ of homozygous ${ }^{\star} 1$ individuals and heterozygosity for ${ }^{\star} 2 /{ }^{\star} 3$ decreases intrinsic clearance, and therefore the required dose to $47 \%$ of that of homozygous ${ }^{\star} 1$ individuals. ${ }^{1}$

A number of other CYP2C9 polymorphisms are available for testing on several assay platforms. However, limited genotypephenotype correlations exist for these polymorphisms. As indicated below, the population distributions of CYP2C9 and VKORC1 genotype vary by ethnicity. ${ }^{17,26,27}$ The degree to which differences in prevalence in different populations will influence the clinical utility of testing within those populations remains unclear.

Although a number of additional identified CYP2C9 alleles are described on The Human CYP Allele Nomenclature Committee Web site (http://www.cypalleles.ki.SE/cyp2c9.htm), less information is available about these, than the aforementioned CYP2C9 alleles. Nevertheless, these should be kept in mind if an individual patient is identified with a low warfarin dosing requirement, but with none of the common CYP2C9 polymorphisms.

$C Y P 2 C 9^{\star} 2$ and ${ }^{*} 3$ are found in the major racial groups, but with different allelic frequencies. These alleles should be tested in all individuals. There are also several rare alleles of CYP2C9 alleles that have different frequencies in different ethnic pop- ulations, and some alleles are preferentially found in only certain racial groups. Some CYP2C9 alleles, such as CYP2C ${ }^{\star} 5$, ${ }^{*} 6$, and ${ }^{*} 11,28$ are preferentially found in African-descendent populations at low allele frequencies, but are not found in Asian-descendant populations. On the other hand, the rare CYP2C $9{ }^{*} 4$ polymorphism has only been reported in individuals from Asia. ${ }^{29}$ The decision to test for polymorphisms other than $\mathrm{CYP} 2 \mathrm{C} 9{ }^{*} 2$ and ${ }^{*} 3$ should be based on the populations being tested by a laboratory and the capability to make patient management decisions informed by these less-frequently encountered alleles.

Studies have addressed the question of whether pharmacogenetic testing might predict the INR itself, or predict the risk for bleeding with warfarin. For example, in retrospective studies, INR values above 3.0 are twice as likely among CYP2C9 heterozygotes (relative risk of 2.0 or higher), and are more likely to occur in the first and second week (induction phase) after warfarin initiation than in the third week or later. ${ }^{30,31}$ It follows that CYP2C9 status is a significant predictor of the INR.

\section{Vitamin K epoxide reductase complex 1 (OMIM 608547)}

Polymorphisms within VKORC1, a target of warfarin inhibition, may explain $23 \%$ of warfarin dosing variability. Warfarin sensitivity haplotypes have been developed for VKORC1. ${ }^{17}$ High-dose (low sensitivity), intermediate-dose (intermediate sensitivity), and low-dose (high sensitivity) haplotypes were developed on the basis of a panel of SNPs within the gene. Two SNPs in particular were found to predict the warfarin dosing (sensitivity) phenotype: a $\mathrm{G} \rightarrow \mathrm{A}$ transition in the VKORC1 promoter polymorphism, at -1639 ; and a $\mathrm{C} \rightarrow \mathrm{T}$ transition in intron 1 at 1173. ${ }^{32}$ These SNPs are in tight linkage disequilibrium, and determination of the genotype for $-1639 \mathrm{G} \rightarrow \mathrm{A}$ or $1173 \mathrm{C} \rightarrow \mathrm{T}$ permits assignment of warfarin sensitivity status. High-dose (low sensitivity) individuals have the $-1639 \mathrm{~B} / \mathrm{B}$ genotype (the $B$ phenotype is also referred to as the $G$ genotype), whereas individuals requiring a low dose (high sensitivity) have the -1639A/A genotype. Warfarin dosing changes informed by $V K O R C 1$ genotypes have been developed. Relative to $V K O R C 1 \mathrm{~A} / \mathrm{B}$ heterozygotes, warfarin dose is increased by $35 \%$ in BB homozygotes and decreased by $32 \%$ in AA homozygotes.

Other SNPs within the VKORC1 haplotypes originally described have not been found to provide additional information. As such, there is no recommendation to test these SNPs. However, in the context of patients who demonstrate warfarin resistance, several mutations in VKORC1 have been reported. These occur infrequently and result in warfarin doses in excess of $80 \mathrm{mg} /$ week. There is no dosing guidance associated with these mutations at this time. Additional research is needed to clarify whether warfarin is the optimal anticoagulant for individuals expressing warfarin resistance, and to associate warfarin dosing with the mutations in VKORC1.

Another issue for genotyping assays is detecting polymorphisms and mutations for which there are no phenotypic information available. These will occur most frequently in tests using DNA sequencing. These "variations of undetermined 
significance" should be reported based on recommendations formulated by the American College of Medical Genetics (ACMG Recommendations for Standards for Interpretation of Sequence Variations) ${ }^{33}$ and in a manner consistent with the CYP nomenclature committee nomenclature (http://www. cypalleles.ki.SE/) when necessary.

It is important to note that the impact of CYP2C9 and VKORC1 on the maintenance dose of warfarin are independent and additive, but not synergistic (i.e., the presence of variants in both genes does have a greater impact on dose than either independently, but there is not a strict arithmetic relationship). Although a number of algorithms have been developed to allow practitioners to quickly find the appropriate maintenance dose for a specific genotypic pattern, these will inevitably evolve over time as there are currently relatively few studies available in which variants in both CYP2C9 and VKORC1 have been tested in the same patients.

The clinical value (clinical validity and utility) of any pharmacogenetic predictive test for an adverse drug event rests on five key measures.

\section{Parameters of clinical validity of a pharmacogenetic test}

1. Clinical sensitivity-Defined as the proportion of individuals with adverse events that have a genotype other than wild type. This is independent of the analytical validity of the test. Insensitive tests may still be valuable if they are specific.

2. Clinical specificity — defined as the proportion of individuals with no adverse events that have the wild-type $\left({ }^{\star} 1 /\right.$ $\left.{ }^{\star} 1\right)$ genotype.

3. Quantitative change in a patient's (relative) risk-What is the magnitude of the increased risk for an adverse event in the variant genotype group relative to the wild-type group (i.e., clinical positive predictive value)? This is the proportion of individuals with a nonwild-type CYP2C9 allele that have an adverse event.

\section{Parameters of clinical utility of a pharmacogenetic test}

4. Quantitative change in risk relative to benefit-Ultimately it is not possible to separate risk from benefit. It is valuable to ask: what change in the risk-to-benefit profile of the drug in question is conferred by using this test? Alternatively, one might ask, what is the magnitude of the increased risk for an adverse event in the variant genotype group relative to the wild-type group, and what change in benefit occurs from the drug in the variant group? Because this involves a comparison of different types of events (adverse events and benefits), it is not appropriate to treat them as the same, and both the number of events and the quality and clinical importance of those events have to be compared in the variant and wildtype groups and effectively communicated to patients, their families where appropriate, and their care providers. In the case of warfarin, a dosage adjustment based on genotype could reduce the risk of an adverse event (e.g., bleeding), but could potentially expose the patient to a higher risk of clotting, which would be characterized as a reduction in the benefit of the drug.

In situations where a large number of treated patients benefit from a drug, a proportionally small decrease in the number of patients who benefit may offset any decrease in the number of adverse events. If genotyping results in a change in dose that reduces the number of adverse events, does it also alter the number of patients who would benefit from the drug? This balance is also affected by the magnitude of the adverse event or benefit. If genotyping resulted in a decrease of clinically significant bleeding events of low morbidity (e.g., hematoma or gum bleeding) but the patients experienced a higher rate of a high morbidity adverse outcomes related to benefit (e.g., pulmonary embolism), this may not be desirable even if the absolute number of bleeding events was more than the number of pulmonary embolisms.

5. The incremental clinical benefit of the pharmacogenetic test-In the clinical setting, it is important to know what incremental benefit any test provides over existing methodologies. Simply put, if there is robust ability to predict an adverse event already using the accepted INR proto$\mathrm{col}$, there would be little incremental value in the development and application of any new test unless it can be shown to be cheaper or more effective in patient management. As more and more effective technologies and biomarkers to predict drug effects become used, this will become more difficult with time.

These key questions can all be applied to the case of pharmacogenetic testing to predict the effects of genotype on warfarin response. It is important to note that the tests for CYP2C9 variants and $V K O R C 1$ variants that have been developed have been studied using three phenotypes: (1) the absolute value of the INR itself, (2) the dose that patients are treated with once they arrive at a stable dose, and (3) the incidence and severity of bleeding events. The last is the only clinical outcome that directly impacts the patient's health and well-being. While the first two phenotypes are technically clinical outcomes, they are best characterized as intermediate outcomes that may relate to the clinical outcome of interest (i.e., bleeding). These phenotypes should be addressed independently unless there are data that suggest that an intermediate outcome (such as stable warfarin dose) is strongly associated with another clinical outcome (such as incidence of bleeding events). The clinical outcome of bleeding is the one for which most data are available, although other clinical outcomes such as increase in thromboembolism associated with a lower dose are important to consider. The application of these criteria to bleeding is outlined below.

\section{Clinical validity}

1. Clinical sensitivity. With variant CYP2C9 genotypes grouped together from two studies, the clinical sensitivity 
of CYP2C9 to identify serious bleeding events is $46 \%$ (95\% confidence interval [CI] 32-60\%), ${ }^{1}$ indicating that about half of all serious bleeding events occur among individuals with wild-type CYP2C9.

2. Clinical specificity. Overall, the clinical specificity of CYP2C9 is 69\% (95\% CI 62-75\%). The correspondingly high false-positive rate (31\%) is because variant CYP2C9 genotypes are relatively common and most will not experience serious bleeding.

3. Relative risk. The relative risk for the adverse event of serious bleeding in individuals with wild versus variant genotypes in these studies is 1.7 (95\% CI $0.8-3.6){ }^{1}$

\section{Clinical utility}

Although observational associations of pharmacogenetic variants and both dose and adverse drug events are of value, they do not themselves inform providers about whether use of this testing is able to reduce the rate of adverse events experienced by patients taking warfarin, while maintaining the efficacy of the drug. That said, the strong association with dose does suggest that pharmacogenetic testing is valuable in the diagnostic setting when patients require very low maintenance doses of warfarin, or when the INR achieved during the induction phase is unusually high. However, in considering the utility of these tests in minimizing the adverse outcomes, alternative methodologies should be considered as well.

4. Attributable risk. The attributable risk expresses the proportion of the adverse events that are attributable to the nonwild-type genotypes. About $7 \%$ of bleeding events among those with a nonwild-type genotype are directly related to that genotype. ${ }^{1}$

5. Risk relative to benefit. No data are available on the influence of pharmacogenetic testing on the efficacy of warfarin. If a patient receives a lower dose of warfarin because they have a variant genotype it is not clear whether or not they would experience an increased chance of embolism, as well as a decreased chance of bleeding. The quantitation of any such increased risk of an embolic event would be required for any assessment of change in risk relative to effect. In the setting of warfarin, this is a situation, where a large number of treated patients benefit from a drug, and where a small decrease in the proportion of patients who benefit may offset any decrease in the number of adverse events. If genotyping results in a change in dose that reduces the number of adverse events, does it also alter the number of patients who would benefit from the drug?

Reliable data on both the decreased chance of bleeding and the increased chance of embolism should be available to make this assessment, and should be presented to the patient separately and clearly. Because each individual patient may view these risks and benefits within the context of his or her own medical condition and societal context, it is not valuable in clinical settings to try to present a generalizable ratio or other algorithm that attempts to mathemati- cally estimate a single overall risk-to-benefit ratio. In the same way as patients should be counseled about drug therapy by informing them about the potential value of the treatment and its potential side effects, so the value of a pharmacogenetic test should be presented with its potential value to improve benefit and reduce risk.

6. Incremental benefit. Since the first studies carried out on pharmacogenetic tests for warfarin, ${ }^{24}$ a significant number have been carried out in specialized anticoagulation clinics where the INR is used consistently. This, together with the number of adverse bleeding events per year that occur despite widespread use of the INR and with the large number of anticoagulation clinics, argues that there is potentially considerable incremental value to be gained from pharmacogenetic testing. The incremental value of this testing in specific clinics, hospitals, and health care settings may well depend on factors such as the availability of anticoagulation clinics, the variability in the INR, and the disease burden and comorbidity of the population being served.

In general, in clinics where patients are followed very closely and the INR is frequently and effectively monitored, the value of additional predictive testing would appear less. In contrast, in more resource-compromised settings or situations where less directed attention is paid, pharmacogenetic testing may be of greater value although there are no studies that address this question. Although a number of attempts to assess the value of pharmacogenetics testing from an economic standpoint have been published, these have limitations, and economic models that assess the incremental value of pharmacogenetic testing in specific environments are not available. This represents a significant gap in knowledge at present.

No prospective study in any setting has yet shown genotyping to be effective in reducing the incidence of high INR values, the time to stable INR, or the occurrence of serious bleeding events. One small pilot randomized trial enrolled 38 patients and found six serious bleeding events among the 20 patients with standard warfarin dosing versus two bleeding events among the 18 receiving model-based dosing. ${ }^{34}$

Larger prospective trials that test whether genotyping is effective in reducing this increased risk of bleeding, in reducing the incidence of high INR values, or in reducing the time to stable INR are not available but are needed.

\section{Other genes and polymorphisms}

- Various research studies have implicated other genes in the variability of warfarin dosing in some patient populations. These include $\gamma$-glutamyl carboxylase, ${ }^{35}$ several clotting factors, including protein C, Factor II, and Factor $\mathrm{VII},{ }^{36}$ genes involved in transporting vitamin $\mathrm{K}$ warfarin, including apolipoprotein E and P-glycoprotein, and additional genes involved in vitamin K-epoxide metabolism. Studies on the contribution of these polymorphic genes to warfarin dosing variability are on-going. Several factors should be considered before offering these in warfarin sensitivity testing. 
- Have the studies been replicated?

- Is the allele frequency for a polymorphism or mutation in these genes sufficiently high enough to warrant testing in all populations or only in a subset of identified populations?

- Is the allelic variation informative for dosing alterations?

- Is there evidence beyond dose prediction of clinical impact?

- What is the incremental cost associated with adding an additional test(s)?

As a general principle, three independent, concordant studies should be available before implementing new testing, and this should be applied to other candidate genes for inclusion in warfarin sensitivity testing (Clinical Laboratory Standards Institute, 2005). Currently, some manufacturers are testing polymorphisms in CYP2C9 with allele frequencies of $\sim 0.005$. It is important that the addition of other gene variants to testing panels be based on strong evidence and it is essential that statistically supported dosing information be associated with the allele to inform patient management dosing alterations.

\section{Gaps in knowledge of clinical validity}

As with analytical validity, the evidence base for clinical performance characteristics of CYP2C9 and VKORC1 testing is poorly organized. As such:

- clinical sensitivity, clinical specificity, relative risk, and attributable risk of severe bleeding in VKORC1 haplotypes and $C Y P 2 C 9$ and $V K O R C 1$ genotypes combined are poorly characterized;

- the contribution of genetic versus other influences toward bleeding is poorly understood for many populations;

- positive and negative predictive values for severe bleeding in the VKORC1 haplotypes and the CYP2C9 and VKORC1 genotypes combined is poorly understood ${ }^{1}$; and

- understanding of the clinical performance characteristics in those with rare alleles and the compound heterozygotes with those alleles is less well informed than is the evidence for the common alleles.

Because CYP2C9 and VKORC1 account for only $30-50 \%$ of the variability in the dosing of warfarin at stable INR, it is important to expand our understanding of the roles of other genes in the pharmacodynamics and pharmacokinetics, as well as the roles of environmental and genetic modifiers, in warfarin metabolism.

\section{Recommendations}

To provide the evidence needed to better understand the clinical performance characteristics of CYP2C9 and VKORC1 testing robust data should be collected on:

- the association between CYP2C9 and VKORC1 genotypes and warfarin at steady state in all ethnic groups for which testing is being considered;

- the role of both genetic and nongenetic factors in the variability of warfarin dosing; and
- the relationship between adverse events and CYP2C9 and VKORC1 genotypes.

\section{Can the reliability of INR measurements be improved and would their improvement be more important or cost-effective than genotyping?}

Warfarin dosing variability is affected by known genetic and nongenetic factors such as drug interactions from concomitant medications and some as yet unknown factors. Successfully maintaining the patient's anticoagulation state within the target range can be improved by better use of informative data from any of the known factors. ${ }^{37}$

The adequacy of warfarin dosing and dose adjustments are determined by monitoring the effect of warfarin on the coagulation factors using a surrogate assay-prothrombin time. Prothrombin in the patient's blood is assessed for its ability to clot using a specific substrate. Endpoint detection may be spectrophotometric or mechanical, depending on the manufacturer and assay kit. Improving the reliability of prothrombin measurements has been an ongoing endeavor for many years. Standardizing the prothrombin tests with well-characterized, sensitive thromboplastin reagents has improved the prothrombin time test in the routine testing laboratory. This test is routinely reported out as the ratio of the patient's prothrombin time to that of a reference population, corrected for the sensitivity of the thromboplastin reagent used.

Not all INR testing is performed in well-standardized laboratories. Point of care tests are used in many institutions. These tests are generally well accepted by clinicians and patients because of the convenience they provide. However, these INR measurements may demonstrate greater variability than the standardized laboratory INR. ${ }^{38}$ Another means of performing INR testing is through home testing devices that have been cleared by the FDA.

Patient education programs and warfarin quality improvement initiatives may reduce the variability of patients' anticoagulation status and these efforts should proceed. However, there is no evidence that these programs alone will be successful at reducing adverse events from relative warfarin overdosing.

Therapeutic drug monitoring for warfarin could be used to augment INR improvements. This has been available since the mid-1970s. The warfarin steady-state concentration is determined by plasma drug analysis and can be used to assess patient compliance and adjust warfarin dosing, if required. However, this testing can only provide appropriate information if the patient is at steady-state, which can be approximated after 5-7 half-lives of drug elimination. The half-life of warfarin is $\sim 1$ week. So a patient would be informatively assessed after a minimum of 5 weeks. However, one effect of the functional ${ }^{*} 2$ and ${ }^{\star} 3$ polymorphisms in CYP2C9 is to increase the half-life of warfarin elimination. Consequently, patients would have to be tested later than 5-7 weeks to be at steady-state. The major issue with this testing is that a significant proportion of serious adverse events occur in the first 6 weeks of warfarin therapy. Although therapeutic drug monitoring may be difficult to im- 
Table 4

Warfarin dosing by VKORC1 and CYP2C9 genotypes

\begin{tabular}{|c|c|c|c|c|}
\hline VKORC1 & $\begin{array}{l}\text { CYP2C } 9 \text { extensive (normal) } \\
\text { metabolizer }\left({ }^{*} 1 /^{*} 1\right)\end{array}$ & $\begin{array}{l}\text { CYP2C9 intermediate } \\
\text { metabolizer }\left({ }^{*} 1 /{ }^{*} 2\right)\end{array}$ & $\begin{array}{l}\text { CYP2C9 slow metabolizer } \\
\left({ }^{*} 1 /{ }^{*} 3,{ }^{\star} 2 /^{*} 2,{ }^{*} 2 /{ }^{*} 3\right)\end{array}$ & $\begin{array}{c}\text { CYP2C } 9 \text { extremely slow } \\
\text { metabolizer }\left({ }^{*} 3 /{ }^{*} 3\right)\end{array}$ \\
\hline Low sensitivity (BB), \% & 140 & 113 & 91 & 63 \\
\hline Medium sensitivity (AB), \% & 100 & 81 & 65 & 46 \\
\hline High sensitivity (AA), \% & 73 & 58 & 47 & 33 \\
\hline
\end{tabular}

plement for warfarin sensitivity testing, it has value for assessing patient compliance in those cases where concern exists whether the patient is actually taking warfarin.

It is unlikely that any given approach to decrease warfarin dosing variability, if used in isolation, will be successful at significantly reducing warfarin-induced adverse events. It is likely that the greatest improvement to patient care would result by going forward in parallel with reducing the variability of INR testing, providing increased education to patients to better home monitor their INRs, and identifying individuals with genetically predisposed warfarin sensitivity.

Awareness of the language of molecular genetics can be an impediment to uptake and clinical application of genetic testing. The nomenclature of genetic variation in the cytochrome P450 system lacks a relationship to functionality to which primary care providers can relate.

Although there is good evidence that the described variants are correlated with the warfarin dose, at present there is insufficient evidence that dosing informed by the variants will alter clinically important outcomes of interest such as major bleeding or subsequent thrombotic events. To be useful, the test results generally need to be available at or near the time of warfarin initiation. In some clinical scenarios, this may require that either results of prior testing be readily available or that point-of-care testing be available. In other rare circumstances, point-of-care testing may be useful but testing systems to do so are not yet widely available.

Recognizing that this testing may be performed, recommendations based on expert consensus from the workgroup about modifications of practice are as follows:

- If used for elective orthopedic patients, test results could be made available before dosing.

- For other indications prompt genotyping also may maximize its clinical utility. Test results may not need to be available at the time of the first warfarin dose, but they should be available within 3 days to allow for dosing adjustments to be made before stabilization of the INR.

- There is no role for VKORC1 testing once stable warfarin dosing has been achieved. There are insufficient data to define the role, if any, of testing CYP2C9 in someone who is already on warfarin. However, the prolonged half-life resulting from $C Y P 2 C 9$ variants might be useful in certain situations yet to be determined.

- At present, there seems to be no role for confirmatory testing for these variants (except in cases where lab error or sample mix-up is suspected).
- Patient reports of pharmacogenetic testing should be linked to algorithms (e.g., http://warfarinDosing.org) that can assist providers in integrating patient variables and test results together to inform adjustments to initial dosing. We have standardized the following dosing adjustments around a percent reduction from starting dose rather than to state-specific doses. These are adjustments to the predicted starting dose based on nongenetic factors as reflected in http://warfarinDosing.org (Table 4). ${ }^{39}$

\section{Gaps in clinical utility knowledge}

In addition to the gaps identified in the analytical and clinical validity section that have implications for clinical utility, significant gaps in knowledge exist that prevent a current recommendation for the widespread use of pharmacogenetic testing to improve the safety of warfarin, while maintaining its efficacy. These include:

- the lack of adequately powered prospective trials that test whether pharmacogenetically guided therapy is able to reduce the risk of warfarin-associated bleeding in the initiation of warfarin therapy, during the maintenance phase, or during longer periods of therapy, in which it is well documented that risk persists ${ }^{40}$;

- the lack of trials that have tested whether dose adjustments resulting from the use of pharmacogenetic testing are associated with changes in the efficacy of warfarin. This is important because dose reduction is well documented to result in lower efficacy. Because warfarin is such an effective drug, small reductions in efficacy may offset small reductions in toxicity achieved by pharmacogenetic testing;

- the lack of comprehensive data on cost or cost-effectiveness as to the use of $V K O R C 1$ testing alone or in combination with CYP2C9;

- examination of the clinically necessary and/or preferred turn-around-time of CYP2C9 and VKORC1 testing as relates to the clinical situations in which it is used;

- a lack of validated educational materials for patients and providers; and

- a lack of guidelines for the evaluation of program performance.

\section{Recommendations}

- Studies should be conducted to assess the efficacy of CYP2C9 and VKORC1 combined testing in the same populations in which the risks of warfarin are quantified. 
- Cost-effectiveness studies of combined CYP2C9 and VKORC1 testing in reducing warfarin related adverse events are needed.

\section{Ethical, legal, and social issues}

Unique ethical, legal and social issues (ELSI) are relatively minimal for this type of testing. The type of information to be communicated in reports must be addressed. In particular, the implications for other family members should be considered.

\section{DISCUSSION}

The use of warfarin involves a nearly half-billion dollar marketplace (Next Generation Pharmaceuticals, 2007) ${ }^{41}$ related to the drug cost and its management and recommendations related to its use should meet the highest standards. There is insufficient evidence in the literature to warrant recommending routine warfarin sensitivity genotype testing before patients receive warfarin therapy. ${ }^{1}$ Outcomes from large prospective clinical trials are needed that link genotype to warfarin dosing recommendations before endorsing warfarin sensitivity genotype testing. Outcomes from these studies should help clarify the benefits, disadvantages, dosing adjustments, and costs associated with this testing and clarify if testing leads to improvements in the quality of patient care. Attention must also be paid to the impact of implementation of testing in different clinical settings, given that the majority of patients are initiated on warfarin outside of academic medical centers and specialized anticoagulation clinics, where the clinical trials are likely to be performed. Genotyping for warfarin sensitivity, however, is clinically available from a number of laboratories.

The clinical validity and utility of pharmacogenetic tests hinges on their analytical validity, but importantly also depends upon how well they impact important clinical outcomes of drug effect. In this respect pharmacogenetic tests differ from conventional genetic tests designed to predict a phenotype of disease. This is because all drugs have side effects. It follows that this kind of test may be designed to predict efficacy or to predict adverse events, or both. The tests addressed here to predict the dose and effects of warfarin might reasonably be used to predict both the efficacy of the drug and its principle adverse effect: unanticipated bleeding episodes. Few data are currently available that allow us to determine whether testing for genetic variants in CYP2C9 and VKORC1 might improve (or least not decrease) the efficacy of warfarin, or whether any reduction in adverse events that we might achieve through more careful warfarin dosing might be balanced by reductions in efficacy. In contrast, a considerable body of evidence has associated these genetic variants with warfarin dose and to a much lesser degree with the incidence of bleeding events during warfarin therapy. On the surface, any tool that might reasonably reduce the considerable morbidity and mortality that all agree is associated with the use of warfarin should be seriously evaluated because of the potential value to patients, their care providers and to health care systems that strive to provide safe and effective care, and are increasingly monitored to ensure they do so.

The evidence available to date and examined during the ACCE Review (See page 89, this issue) concluded that strong evidence is available for the clinical validity of testing both the CYP2C9 and VKORC1 to predict stable warfarin dose. This is an important, but intermediate outcome, one step removed from the outcome of interest of the review-bleeding risk. In contrast, the evidence seems weak for any association between CYP2C9 testing and severe bleeding events (clinical sensitivity $46 \%$ [95\% CI 32-60\%]; specificity 69\% [95\% CI 62-75\%]). This may reflect the impact of multiple other factors such as diet and age, as well as factors that are currently unknown to alter bleeding risk. No such data are available for VKORC1 testing.

\section{A research agenda informed by the gaps in knowledge}

As discussed under Methods/Process, the decision was to not grade the evidence on which the following recommendations are made because of the lack of evidence in several critical areas. Gaps in knowledge inform a research agenda that can help to resolve the uncertainties identified. The breadth and complexity of the gaps to be filled suggest that a large randomized prospective clinical trial that addresses the relationship between genotypes and all clinical outcomes from warfarin treatment is needed. Important considerations in the development of such a trial include defining primary and secondary clinical endpoints and trial design. Issues that will have to be considered include privacy, structured data elements, followup, who submits data, who would access data, what questions the data address, where the data would reside, and who maintains and updates the data. There may be roles for PharmGKB, a federal program and Web site that curates information that establishes knowledge about the relationships among drugs, diseases, and genes, including their variations and gene products and the Centers for Medicare and Medicaid Services in addressing the prospective data collection.

\section{Overarching summary and recommendations}

In the context of variable warfarin sensitivity, there is limited evidence at this time to support routine testing of the CYP2C9 and VKORC1 genes for functional polymorphisms that affect warfarin dosing. Although the analytic testing is currently being performed in a number of laboratories, there is less linkage of the genotype data produced with phenotypic warfarin dosing than is optimal for the development of recommendations for clinical practice.

- There are no prospective data to recommend for or against routine CYP2C9 and VKORC1 testing in warfarinnaive patients because no substantive prospective study has yet shown this intervention to be effective in reducing the incidence of high INR values, the time to stable INR, or the occurrence of serious bleeding events, while maintaining the ability of the drug to prevent thromboembolic events. 
- CYP2C9 and VKORC1 genotypes can reasonably be used as part of diagnostic efforts to determine the cause of an unusually low maintenance dose of warfarin or an unusually high INR during standard dosing. In doing so, health care providers would be able to spend less time and energy on the issues of drug interactions and diet, both of which can be inordinately time consuming in this setting.

- CYP2C9 testing beyond ${ }^{\star} 2$ and ${ }^{*} 3$ alleles involves rare alleles for which there is much more limited data available to support their inclusion.

\section{ACKNOWLEDGMENTS}

We are grateful to Annette Taylor, $\mathrm{PhD}$ and Matthew Taylor, MD, for their prepublication reviews of this position statement. We are grateful to Mark Linder, PhD and Nita Limdi, PharmD for presenting unpublished information to our work group. We are particularly grateful to Monica McClain, $\mathrm{PhD}$, and Glenn Palomaki for their development of their RapidACCE review and for reviews of this manuscript. Lastly, we are grateful to Lawrence Lesko, $\mathrm{PhD}$ for his participation in our meeting. This work group was partially supported by funds from TM Biosciences and the American College of Medical Genetics Foundation to which we are grateful.

\section{References}

1. McClain M, Palomaki G, Piper M, Haddow JE. A Rapid-ACCE review of CYP2C9 and $V K O R C 1$ allele testing to inform warfarin dosing in adults at elevated risk for thrombotic events to avoid serious bleeding. Genet Med 2008;10:89-98.

2. Albers GW, Amarenco P, Easton JD, Sacco RL, et al. Antithrombotic and thrombolytic therapy for ischemic stroke: the Seventh ACCP Conference on Antithrombotic and Thrombolytic Therapy. Chest 2004;126:483S-512S.

3. Büller HR, Agnelli G, Hull RD, Hyers TM, et al. Antithrombotic therapy for venous thromboembolic disease: the Seventh ACCP Conference on Antithrombotic and Thrombolytic Therapy. Chest 2004;126:401S-428S. Erratum: Chest 2005;127:416.

4. Harrington RA, Becker RC, Ezekowitz M, Meade TW, et al. Antithrombotic therapy for coronary artery disease: the Seventh ACCP Conference on Antithrombotic and Thrombolytic Therapy. Chest 2004;126:513S-548S.

5. Hylek E, Antonio J, Molina C, Shea C, et al. Translating the results of randomized trials into clinical practice. The challenge of warfarin candidacy among hospitalized elderly patients with atrial fibrillation. Stroke 2006;37:1075-1080.

6. Wittkowsky A. Effective anticoagulation therapy: defining the gap between clinical studies and clinical practice. Am J Manag Care 2004;10:S297-S306.

7. Higashi MK, Veenstra DL, Kondo LM, Wittkowsky AK, et al. Association between CYP2C9 genetic variants and anticoagulation-related outcomes during warfarin therapy. JAMA 2002;287:1690-1698.

8. Sanderson S, Emery J, Higgins J. CYP2C9 gene variants, drug dose, and bleeding risk in warfarin-treated patients: a HuGEnet systematic review and meta-analysis. Genet Med 2005;7:97-104.

9. Wadelius M, Sörlin K, Wallerman O, Karlsson J, et al. Warfarin sensitivity related to CYP2C9, CYP3A5, ABCB1 (MDR1) and other factors. Pharmacogenomics J 2004;4:40-48.

10. Rost S, Fregin A, Ivaskevicius V, Conzelmann E, et al. Mutations in VKORC1 cause warfarin resistance and multiple coagulation factor deficiency type 2. Nature 2004; 427:537-541.

11. Loebstein R, Dvoskin I, Halkin H, Vecsler M, et al. A coding VKORC1 Asp36Tyr polymorphism predisposes to warfarin resistance. Blood 2007;109:2477-2480.

12. Reynolds KR, Valdes R Jr, Hartung BR, Linder MW. Individualizing warfarin therapy. Personal Med 2007;4:11-31.

13. Linder MW, Looney S, Adams JE 3rd, Johnson N, et al. Warfarin dose adjustments based on CYP2C9 genetic polymorphisms. J Thromb Thrombolysis 2002;14:227-232.

14. King B, Khan TI, Aithal G, Kamali F, et al. Upstream and coding region CYP2C9 polymorphisms: correlation with warfarin dose and metabolism. Pharmacogenetics 2004;14:813-822.

15. Gudgeon JM, McClain MR, Palomaki GE, Williams MS. Rapid ACCE: experience with a rapid and structured approach for evaluating gene-based testing. Genet Med 2007;9:473-478.
16. Haddow JE, Palomaki GE. ACCE: a model process for evaluating data on emerging genetic tests. In: Khoury MJ, Little J, Burke W, editors. Human genome epidemiology: a scientific foundation for using genetic information to improve health and prevent disease. Oxford: Oxford University Press, 2003.

17. Rieder MJ, Reiner AP, Gage BF, Nickerson DA, et al. Effect of VKORC1 haplotypes on transcriptional regulation and warfarin dose. N Engl J Med 2005;352:2285-2293.

18. Palomaki GE, Haddow JE, Bradley LA, Richards CS, et al. Estimated analytic validity of HFE C282Y mutation testing in population screening: the potential value of confirmatory testing. Genet Med 2003;5:440-443.

19. Palomaki GE, FitzSimmons SC, Haddow JE. Clinical sensitivity of prenatal screening for cystic fibrosis via CFTR carrier testing in a United States panethnic population. Genet Med 2004;6:405-414.

20. American College of Medical Genetics Standards and Guidelines for Clin Genet Laboratories, 2006. Available at: http://www.acmg.net/Pages/ACMG_Activities/ stds-2002/stdsmenu-n.htm. Accessed October 1, 2007.

21. Clinical Laboratory Standards Institute (CLSI). Molecular diagnostic methods for genetic disease; approved guidelines CLSI document MM01-A2. Wayne, PA: Clinical and Laboratory Institute, 2006.

22. Organization for Economic Co-Operation and Development. Guidelines for quality assurance in molecular genetic testing, 2007. Available at: http://www.oecd.org/ document/24/0,3343, en_2649_34537_1885208_1_1_1_1,00.html Accessed October 1, 2007.

23. National Academy of Clinical Biochemistry. Draft guidelines and recommendations for laboratory analysis and application of pharmacogenetics to clinical practice, 2006. Available at: http://aacc.org/AACC/members/nacb/LMPG/OnlineGuide/ DraftGuidelines/Pharmacogenetics/ Accessed October 1, 2007.

24. Aithal GP, Day CP, Kesteven PJ, Daly AK. Association of polymorphisms in the cytochrome P450 CYP2C9 with warfarin dose requirement and risk of bleeding complications. Lancet 1999;353:717-719.

25. Millican EA, Lenzini PA, Milligan PE, Grosso L, et al. Genetic-based dosing in orthopedic patients beginning warfarin therapy. Blood 2007;110:1511-1515.

26. Lee CR, Goldstein JA, Pieper JA. Cytochrome P450 2C9 polymorphisms: a comprehensive review of the in-vitro and human data. Pharmacogenetics 2002;12:251-263.

27. Imai J, Ieiri I, Mamiya K, Miyahara S, Furuumi H, et al. Polymorphism of the cytochrome P450 (CYP) 2C9 gene in Japanese epileptic patients: genetic analysis of the CYP2C9 locus. Pharmacogenetics 2000;10:85-89.

28. Tai G, Farin F, Rieder MJ, Dreisbach AW, et al. In-vitro and in-vivo effects of the CYP2C $^{\star} 11$ polymorphism on warfarin metabolism and dose. Pharmacogenet Genomics 2005;15:475-481.

29. LeeSY, Nam MH, Kim JS, Kim JW. A case report of a patient carrying CYP2C $9 * 3 / 4$ genotype with extremely low warfarin dose requirement. J Korean Med Sci 2007;22:557-559.

30. Lindh JD, Lundgren S, Holm L, Alfredsson L, et al. Several-fold increase in risk of overanticoagulation by CYP2C9 mutations. Clin Pharmacol Ther 2005;78:540-550.

31. Peyvandi F, Spreafico M, Siboni SM, Moia M, et al. CYP2C9 genotypes and dose requirements during the induction phase of oral anticoagulant therapy. Clin Pharmacol Ther 2004;75:198-203.

32. Reitsma PH, van der Heijden JF, Groot AP, Rosendaal FR, et al. A C1173T dimorphism in the VKORC1 gene determines coumarin sensitivity and bleeding risk. PLoS Med 2005;2:e312.

33. ACMG recommendations for standards for interpretation of sequence variations, 2006. Available at: http://www.acmg.net/resources/policies/pol-027.pdf Accessed October 1, 2007.

34. Hillman MA, Wilke RA, Yale SH, Vidaillet HJ, et al. A prospective, randomized pilot trial of model-based warfarin dose initiation using CYP2C9 genotype and clinical data. Clin Med Res 2005;3:137-145.

35. Wadelius M, Chen LY, Downes K, Ghori J, et al. Common VKORC1 and GGCX polymorphisms associated with warfarin dose. Pharmacogenetics J 2005;5:262-270.

36. Shikata E, Ieiri I, Ishiguro S, Aono $\mathrm{H}$, et al. Association of pharmacokinetic (CYP2C9) and pharmacodynamic (factors II, VII, IX, and X; proteins S and C; and gamma-glutamyl carboxylase) gene variants with warfarin sensitivity. Blood 2004; 103:2630-2635.

37. Gage BF, Eby C, Milligan PE, Banet GA, et al. Use of pharmacogenetics and clinical factors to predict the maintenance dose of warfarin. Thromb Hemost 2004;91:87-94.

38. McBane RD 2nd, Felty CL, Hartgers ML, Chaudhry R, et al. Importance of device evaluation for point-of-care prothrombin time international normalized ratio testing programs. Mayo Clin Proc 2005;80:181-186.

39. Warfarin dosing, 2006. Available at: http://WarfarinDosing.org Accessed October 1, 2007.

40. Limdi NA, McGwin G, Goldstein JA, Beasley TM, et al. Influence of CYP2C9 and VKORC1 1173C/T genotype on the risk of hemorrhagic complications in AfricanAmerican and European-American patients on warfarin. Mol Ther 2008;83:312321.

41. Next generation pharmaceuticals, 2007. Available at: http://www.'ngpharma.com/ pastissue/article.asp art $=269086$ \&issue $=185^{\prime} .2007$ Accessed October 1, 2007. 